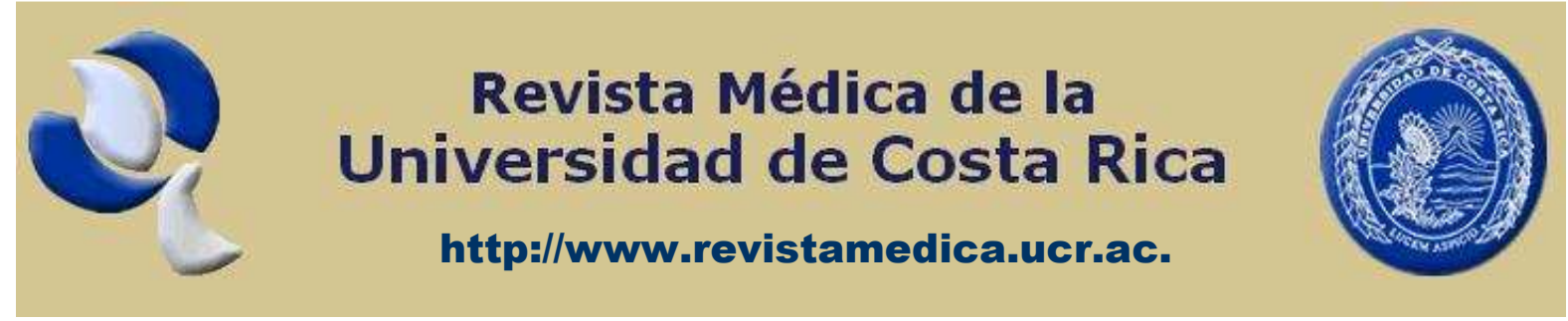

Sección Estudiantil

\title{
Tratamiento con Manzanilla (Matricaria chamomilla), para reducción de las ojeras.
}

\author{
Jiménez Delgado, Jefrey'; Madrigal Rojas, José ${ }^{1}$ y Salazar Barrantes, Sofía'.
}

1. Estudiantes de la Escuela de Medicina de la Universidad de Costa Rica, San Pedro, San José, Costa Rica.

\section{Resumen:}

Objetivo: Estudiar el efecto de la manzanilla en las ojeras y en la inflamación en la zona periocular. Metodología: Estudio en paralelo $(n=15)$, se aleatorizó los sujetos en una proporción 2:1 al tratamiento tópico o control respectivamente. Para medir los resultados, se usó una escala de tonalidad utilizando Macromedia Fireworks y se aplicó una encuesta a todos los sujetos en estudio.

El análisis estadístico se realizó con el software Epi Info® versión 3.3. Se analizaron los porcentajes y se realizó un Test de Fisher con cálculo de OR con el objetivo de detectar una disminución de 4 tonos en la escala.

Resultados: El 70\% de los individuos del grupo intervención consideraron efectivo el tratamiento en la disminución de la tonalidad y volumen de las ojeras; el $80 \%$ sintió mayor relajación en la región periocular. Se obtuvo un $\mathrm{OR}=16$; IC 95\%= $(0.75-760.46)$; $\mathrm{p}=0.046$ para la disminución de la tonalidad de las ojeras.

Conclusiones: No se encontró diferencia estadísticamente significativa en la disminución de las ojeras, sin embargo se observa una tendencia a su disminución.

Palabras clave: Manzanilla - Matricaria chamomilla - Ojeras

Recibido: Enero 2009. Aceptado: Febrero 2009. Publicado: Marzo 2009.

Revista electrónica publicada por el Departamento de Farmacología de la Escuela de 


\begin{abstract}
:
Objective: Study the effect of the chamomile in the dark rings under the eyes and in the swelling in the periocular zone.

Methodology: Study in parallel $(n=15)$, subjects were randomized to topical treatment or control in a proportion 2:1 respectively. To measure the results, was used a scale of tonality using Macromedia Fireworks and a test to all the subjects in study.

The statistical analysis was using the software Epi Info® version 3.3. The data was analyzed with percentages and with a Test of Fisher with estimate of OR.

Results: $70 \%$ of the individuals of the group intervention considered effective the treatment, as regards the tonality and volume of the dark rings under the eyes, $80 \%$ felt main relaxation in the periocular zone. Obtained OR=16; Cl 95\% $=(0.75-760.46) ; \mathrm{p}=$ 0.046 for the reduction of the tonality of the dark rings under the eyes.

Conclusions: No statistical significant difference was found in the decrease of the dark rings under the eyes; however a decrease is observed.
\end{abstract}

Keywords: Chamomille - Matricaria chamomilla - Dark rings under the eyes

\section{Introducción}

La manzanilla tiene constituyentes activos como el levomenol, la matricina y el bisabolol, los cuales le brindan ciertas propiedades medicinales, como su acción antiinflamatoria [1]. Los Flavonoides (como quercetol, apigenina y luteolina) se han asociado con propiedades antiinflamatorias al inhibir la formación y salida de histamina [2]. También posee cumarínicos (como chamillina), los cuales se relacionan con el incremento de los efectos anticoagulantes en pacientes bajo tratamiento con Warfarina [3].

Se ha estudiado el efecto antiinflamatorio de la planta, en la piel y se recomienda el uso de manzanilla para el tratamiento de problemas en el ojo como irritación, chalazión, e inflamación en la zona ocular [4]. En ciertos casos se presentan reacciones alérgicas en la piel, membranas mucosas y los ojos, especialmente al aplicarse en personas alérgicas al polen 0 que tienen hipersensibilidad a plantas de la familia asteracea [1]. La actividad alérgica que ocasiona esta planta no es muy frecuente y raramente es grave [5].

Las ojeras generalmente son producto de retención de líquido en la zona ocular, un exceso de pigmentación o por la congestión vascular en la dermis. También se da por deshidratación, falta de descanso y consumo de drogas, ya que esto produce estrés en el organismo y genera inflamación en los párpados. También se han asociado con acumulación de tejido adiposo bajo los párpados, herencia, personas alérgicas y también individuos con hipertiroidismo, problemas cardíacos o inmunológicos [6].

\section{Métodos y materiales}

Se realizó un estudio aleatorizado, sin ciego, con control, con intervención en paralelo. Se uso una muestra de 15 estudiantes; hombres y mujeres, entre los 18 y 20 años, que

Revista electrónica publicada por el Departamento de Farmacología de la Escuela de 
cursaban el segundo año de la carrera de Medicina en la Universidad de Costa Rica.

Se compró la planta de manzanilla en el mercado central de San José. La infusión se realizó con 6 gramos de flores de manzanilla en 1 litro de agua, se hirvió por 5 minutos y se dejó enfriar a temperatura ambiente. Para medir la cantidad de manzanilla utilizada se uso una balanza digital (marca A\&D modelo GR-200). El tratamiento y el control (agua) se colocaron en recipientes idénticos (frascos de laboratorio de $100 \mathrm{ml}$ ). De día por medio se les dio dos compresas de gasa (Migasa) y el tratamiento ó el agua.

El tratamiento consistía en aplicarse la infusión de manzanilla ó el agua, alrededor de los ojos cerrados, con la gasa, por $15 \mathrm{~min}$, por 7 noches. Previo a la aplicación se debía limpiar la zona con agua. La manzanilla se le aplicó a 10 personas y el control se le dio a 5 personas.

Se excluyó a personas con hipertiroidismo, problemas cardíacos o inmunológicos, con alergia a plantas de la familia asteraceae o al polen.

- Medición de las variables:

Se tomaron fotos del rostro de cada persona con una cámara Sony Cyber-shot DSC W130 (8.1 megapixeles), antes y después del tratamiento y se utilizó una escala de tonalidad (figura 1) para observar el cambio de las ojeras de cada persona; esto se determinó midiendo el grado de pigmentación de las ojeras (según la escala) al inicio y al final y con esto saber cuánto disminuyeron las ojeras en cada persona.
Figura 1. Escala de variación de tonalidad de las ojeras con la aplicación tópica de manzanilla (Matricaria chamomilla) o agua en la zona periocular

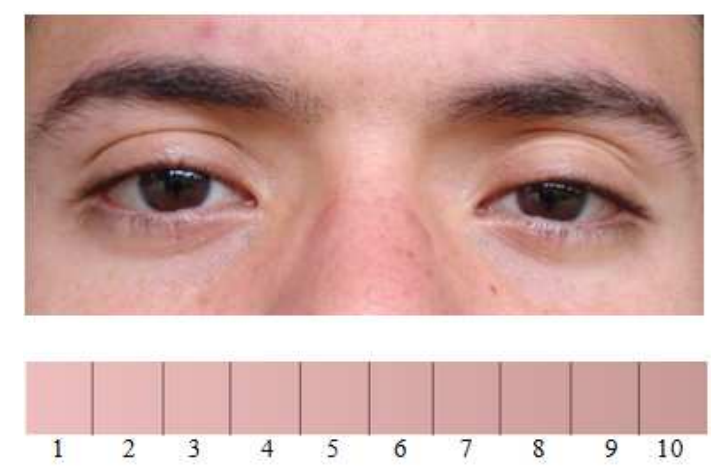

Se realizó una escala para cada persona por medio del software Macromedia Fireworks, con este se tomó la coloración de la ojera (grado más oscuro) y de la frente (grado más claro) y se obtuvo un gradiente en 10 celdas, del mismo tamaño. Se tomó como resultado efectivo, si se tenía una diferencia de 4 o más el grados de tonalidad entre las fotos de antes y después del tratamiento, o sea, que de la escala original, la reducción de por debajo de 7 (figura 1).

Además, a las personas se les aplicó una encuesta con el fin de medir la opinión de cada uno con respecto a la disminución de la tonalidad, inflamación debajo de los ojos, sensación de relajación palpebral, irritación periocular, incomodidad del tratamiento y adherencia.

Para el análisis estadístico de los resultados, se utilizó el software Epi Info® versión 3.3. Se realizó un análisis porcentual y un Test de Fisher con cálculo de OR. 


\section{Resultados}

Cuadro 1. Resultados de la efectividad del tratamiento en el grupo control y en el grupo intervenido con manzanilla (Matricaria chamomilla).

\begin{tabular}{|c|c|c|}
\hline $\begin{array}{c}\text { Característica } \\
\text { evaluada }\end{array}$ & $\begin{array}{c}\text { Efectividad } \\
\text { en el grupo } \\
\text { control (\%) }\end{array}$ & $\begin{array}{c}\text { Efectividad } \\
\text { en el grupo } \\
\text { intervenido } \\
(\%)\end{array}$ \\
\hline $\begin{array}{c}\text { Reducción de } \\
\text { Tonalidad de } \\
\text { las ojeras }\end{array}$ & 20 & 70 \\
$\begin{array}{c}\text { Disminución de } \\
\text { Volumen de la } \\
\text { zona ocular }\end{array}$ & 20 & 70 \\
$\begin{array}{c}\text { Relajación de } \\
\text { la zona ocular }\end{array}$ & 20 & 80 \\
\hline
\end{tabular}

Cuadro 2. Resultados de la efectividad, en la reducción de tonalidad, con la medición fotográfica realizada en los individuos del estudio según la aplicación del tratamiento asignado.

\begin{tabular}{|l|c|c|}
\hline \multicolumn{1}{|l|}{} & Efectivo * $^{*}$ & No efectivo \\
\hline Manzanilla & 8 & 2 \\
Agua & 1 & 4 \\
\hline
\end{tabular}

*Efectivo: reducción de la tonalidad en 4 o más grados según la escala.

Se obtuvo un OR = 16; con un intervalo de confianza (IC) del $95 \%=$ $(0.75$ - 760.46) con una $p$ (Prueba Fisher) $=0.046$

\section{Discusión}

De los resultados de la encuesta, obtuvimos que la mayoría de los sujetos consideraron que el tratamiento sí era efectivo.

La mayoría de los individuos del grupo control no mostraron mejoría alguna. Sin embargo, uno de los individuos sí presento mejoría con el agua, posiblemente porque el sujeto presentaba deshidratación en el tejido antes del tratamiento [7].

En cuanto a los resultados obtenidos con la escala de tonalidad, el OR nos da una fuerte asociación entre la manzanilla y la reducción en el tono de las ojeras. Sin embargo, las pruebas de validación hacen estadísticamente el estudio, no significativo.

Esta inconsistencia pudo darse por tener una muestra control más pequeña que la de Intervención, lo cual se hubiera evitado con un grupo control más grande.

De los dos sujetos que utilizaron manzanilla y no se observó mejoría, uno presentó una irritación conjuntival y palpebral leve debido a una mala aplicación del tratamiento; el otro sujeto no se aplicó el tratamiento 3 días.

\section{Agradecimientos}

Al laboratorio de Bioquímica de la Escuela de Medicina de la Universidad de Costa Rica por el prestamos de instrumentos para las mediciones.

A Fernando Salazar Alvarado por la colaboración con la estadística en la investigación.

A Pablo Álvarez Aguilar por su colaboración.

Revista electrónica publicada por el Departamento de Farmacología de la Escuela de Medicina de la Universidad de Costa Rica, 2060 San José, Costa Rica. ® All rights reserved. 


\section{Referencias}

1. Thornfeldt C. Cosmeceuticals Containing Herbs: Fact, Fiction, and Future. Dermatol Surg 2005;31:873880.

2. Ross S.M. Chamomile: A Spoonful of Medicine. Holist Nurs Pract 2008;22(1):56-57.

3. Rodríguez-Fragoso L., Reyes-Esparza J., Burchiel S.W. Risks and benefits of commonly used herbal medicines in Mexico. Toxicology and Applied Pharmacology 2008;227:125-135.

4. Fraunfelder F.W. Ocular Side Effects From Herbal Medicines and Nutricional Supplements. American Journal of Ophthalmology 2004;138(4):639-647.

5. Rodríguez-Serna M., Sánchez-Motilla M., Ramón R., Aliaga A. Allergic and systemic contact dermatitis from Matricaria chamomilla tea. Contact Dermatitis 1998;39:192-209.
6. Van Stappen, C. Lleva la rinitis al asma? Sociedad Española de Alergología e Inmunología Clínica 2005;(13), 6-13.

7. Anderson, R. Dyspnea and antiplatelet drugs: Little cause for concern with clopidogrel. Thromb Haemost 2008; 517-8.

\section{Información de los autores}

Jefrey Jiménez Delgado. Estudiante de medicina, Universidad de Costa Rica. andreij911@hotmail.com. 8997-6956.

José Pablo Madrigal Rojas. Estudiante de medicina, Universidad de Costa Rica. pablillo28589@hotmail.com. 8892-7403.

Sofía Salazar Barrantes. Estudiante de medicina, Universidad de Costa Rica. sofro saltes@hotmail.com. 8868-1850. 